\title{
Case Report \\ Primary angiosarcoma: A rare tumour of the breast
}

${ }^{1}$ Thaha MM, ${ }^{1}$ Gamage R, ${ }^{1}$ Thameem MM, ${ }^{1}$ Abeysinghe AAG, ${ }^{1}$ Perera BL

\begin{abstract}
Primary angiosarcoma of the breast is a rare neoplasm arising from stromal tissues and accounting for only $0.04 \%$ of primary breast tumours ${ }^{1}$ Atypical clinical presentations and equivocal radiological findings make the diagnosis challenging. Diagnosis by aspiration cytology is difficult ${ }^{2}$. Therefore, macrobiopsy and ultrastructural examination with immunostaining will confirm the diagnosis ${ }^{3}$. It carries a poor prognosis due to rapid growth and haematogenous spread $^{4}$. Surgical extirpation remains the main mode of treatment and adjuvant chemo and immunotherapy are beneficial in some cases ${ }^{1}$. We present a case which was managed with surgery. Key words; Angiosarcoma, Core biopsy, Immunostaining, Adjuvant therapy.
\end{abstract}

\section{Background}

Though it is a rare neoplasm of the breast, because of its rapid growth and poor prognosis, we thought of presenting this case. Here we wanted to highlight the early diagnosis and proper surgical management of this condition.

\section{Case report}

A 72 years old woman presented with a painless lump in right breast for three months. Except for the age and gender she had no risk factors for breast cancer. She had not undergone any significant chest radiation. On examination, a firm, non-tender, $4 \times 5 \mathrm{~cm}$ lump in the upper outer quadrant of the right breast found. It did not have skin or deeper structures attachment. There were no nipple changes or axillary lymphadenopathy. Left breast examination was normal. Breast ultra sound showed a suspicious lesion in right breast. Mammogram revealed an ill-defined, nonspeculated mass, $6 \mathrm{~cm}$ i $\mathrm{n}$ diameter without micro calcification in right breast. Fine needle aspiration cytology (FNAC) was blood stained and contained malignant cells (C5). Repeated core biopsies were only blood stained and no solid materials found. Right modified radical mastectomy with level II axillary clearance was performed as patient preferred mastectomy over breast conservative surgery. Cut section of the specimen revealed a cystic tumour measuring $3 \times 3 \times 2 \mathrm{~cm}$ in the upper outer quadrant with haemorrhage (Figure 1).

${ }^{1}$ Colombo South Teaching Hospital, Kalubowila.
Microscopically, sinusoidal patterns of papillary formation and inter communicating vascular channels lined by proliferating endothelium with atypia were seen (Figure 2-A,B). Immunostaining was positive (Figure 2-C). All lymph nodes were negative of tumour and the surgical margins were tumour free. The diagnosis of grade II angiosarcoma of the breast was made. Abdominal ultra sonography and computed tomography of the chest did not show any metastasis. No adjuvant therapy was decided atoncological reviewand she was followed up regularly. She is actively followed up for three years with biannual clinical and

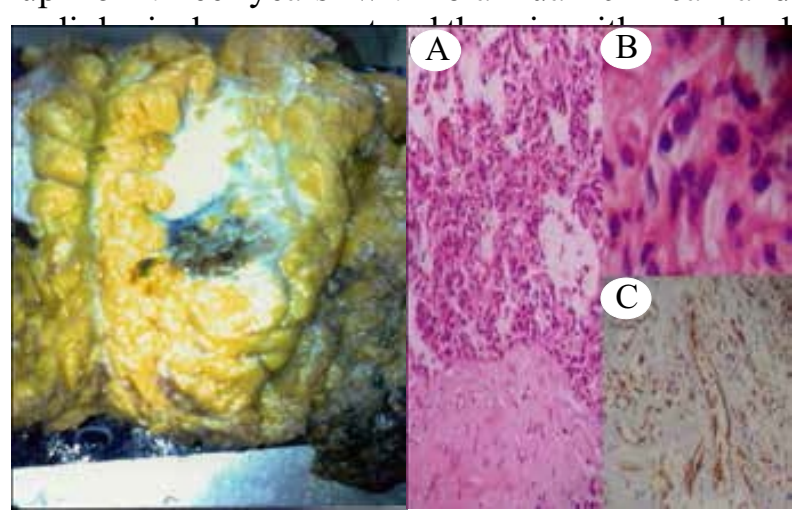

Figure- 1

Figure- 2

Figure 1: Cut Section of the specimen revealing cystic tumour, Figure 2 A, B : sinusoidal patterns of papillary formation and inter communicating vascular channels lined by proliferating endothelium with atypia, Figure $2 \mathrm{C}$ : Positive immunostaining.

\section{Discussion}

Incidence of angiosarcoma among breast sarcoma varies from $2.7 \%$ to $9.1 \%{ }^{2}$. Breast angiosarcoma may develop as a primary neoplasm or more commonly, secondary to breast radiation following breast conservation ${ }^{1}$. The incidence of primary tumour is about 17 new cases permillion women ${ }^{1}$. These tumours occur primarily in young women with $6 \%$ to $12 \%$ of the cases found during pregnancyimplying a hormonal effect ${ }^{1}$ Index patient is older to develop this tumour. It may have an insidious onset, presenting as a painless, discrete mass that grows rapidly ${ }^{3}$. Nipple retraction, discharge or axillary node enlargement is generally absent. In most reported cases, the tumour size is $>$ $4 \mathrm{~cm}$ in diameter ${ }^{3} .17 \%$ of cases may present with a 
bluish discolouration or bruising of the overlying skin $^{3}$. Main mode of spread is haematogenous and lung, skin and subcutaneous tissue, bone, liver, brain and ovary are the common 20 Jaffna Medical Journal, February 2016 sites, in order of frequency ${ }^{2}$. Index case had only painless lump without nipple or skin changes and her metastatic screening was negative.

In most cases, absence of pathognomonic characteristics specific to angiosarcoma will result in a wrong or delayed diagnosis ${ }^{1}$. Sonography usually shows a solid mass that may have well defined or lobulated margins with both hypo and hyperechoic appearance. ${ }^{5}$. On mammogram, they appear as ill defined mass and lack speculation ${ }^{5}$. Breast ultrasound of the index case was inconclusive and mammogram was revealed an illdefined mass without speculation. Preoperative diagnosis by FNAC is difficult and false negative rate of biopsy is $17 \%{ }^{2}$. So, sufficient tumour sampling is necessary to render a final diagnosis, but it is often difficult to perform due to its vascular nature ${ }^{1}$. Core biopsy of the index case did not revealed solid material for the diagnosis.

Complete excision is the best course of action and total mastectomy is the preferable option ${ }^{6}$. However, wide local excision is considered if diameter is $<5 \mathrm{~cm}^{7}$. Since, haematogenous spread is more likely, axillary node dissection is not indicated $^{6}$. In the index case, mastectomy with axillary clearance was performed as there was no pre-operative tissue diagnosis and is what patient also preferred. Ultrastructural examination reveals the vascular nature of angiosarcoma ${ }^{3}$. Pathological

\section{References}

1. Desbiens C, Hogue JC, Levesque Y. Primary breast angiosarcoma: Avoiding a common trap. Case reports in oncological medicine. 2011;2011:517047.

2. Chen KTK, Kirkeggard DD, Bocian JJ. Angiosarcoma of the breast. Cancer. 1980; 46:368-71.

3. Ohta M, Tokuda Y, Kuge S, Okumura A, Tanaka $\mathrm{M}$ et al. A case of angiosarcoma of the breast. Japanese journal of clinical oncology. 1997; 27;91-5.

4. Rosen PP, Kimmel M, Ernsberger D. Mammary angiosarcoma: The prognostic significance of tumour differentiation. Cancer. 1988; 62:214551.

5. Glazebrook KN, Morton MJ, Reynolds C. Vascular tumours of the breast: Mammographic, sonographic and MRI appearances. American journal of roentgenology. 2005; 184:331-8. grading ranges from well differentiated grade I tumours consisting infiltrating bland vascular channels to poorly differentiated grade III with a sarcomatous spindle cell pattern ${ }^{8}$. Immunostaining for factor VIII and CD31 positivity will confirm the diagnosis ${ }^{3}$. Immunostaining for CD31 in the index case was positive.

The prognosis depends on tumour grade, tumour size at diagnosis and margin status at surgery ${ }^{9}$. Five years disease free survival rate for low grade tumours can be as high as $78 \%$ and upto $70 \%$ for grade IItumours, whereas it is only $15 \%$ for higher grade $^{4}$. Because, breast angiosarcoma is very rare, there is no established standard treatment ${ }^{3}$. Mastectomy and chemotherapy is the most likely treatment. However, the role of adjuvant chemotherapy is ill defined, because of rarity of this tumour and lack of prospective studies. ? Chemotherapy might have a greater efficacy in high grade tumours? ${ }^{7}$ but, it is of minimal benefit for disseminated disease $\mathrm{s}^{4}$. The tumour is refractory even after systemic chemotherapy ${ }^{1}$.Indexpatient had grade II tumour without any metastasis, she had not been started on any adjuvant therapy, but kept under active surveillance. Angiosarcomas are relatively resistant toradiotherapy'. Some authors recommend adjuvant radiation when surgical margins are less than $2 \mathrm{~cm}^{1}$. Hormonal treatment is inappropriate since, these tumours usually do not express hormone receptors ${ }^{1}$. Immunotherapy with monoclonal antibodies which have affinity to tumour vasculature antigen-Endoglin are also used in the treatment but they are at research level 3 . Although the literature reveals breast angiosarcoma as a malignancy of poor prognosis, index patient is fortunate not to have local recurrence upto 3 years of follow up.

6. Adem C, Reynolds C, Ingle JN, Nascimento AG. Primary breast sarcoma: Clinicopathologic series from the Mayo clinic and review of the literature. British journal of cancer. 2004; 91:237-41.

7. Zelek L, Llombart-Cussac A, Terrier P et al. Prognostic factors in primary breast sarcoma: A series of patients with long term follow up. Journal of clinic al oncology. 2003;21:2583-8.

8. Donnel RM, Rosen PP, Lieberman PH, Kaufman RJ, Kay S et al. Angiosarcoma and other vascular tumours of the breast.American journal of surgical pathology. 1981; 7:629-42.

9. Johnson CM, Garguilo GA. Angiosarcoma of the breast: A case report and literature review. Current surgery. 2002; 59:490-4. 